\title{
45 Jahre Musiklesesaal (1970 - 2015)
}

Die Musiksammlung der WLB ist die größte in Baden-Württemberg und "gehört zu den großen der Bundesrepublik" - so wird schon im jüngeren der beiden Bibliotheksführer, in denen der Musiklesesaal und die Musiksammlung kompakt und doch umfassend beschrieben sind, berichtet. Bereits seit 1879 wurde der Wunsch nach einer eigenen Musikabteilung sowohl von Benutzern als auch von der Bibliothek dem Unterhaltsträger gegenüber immer wieder vorgetragen. Doch bis zur Realisierung sollte es noch fast 100 Jahre dauern. Erst für den 1970 fertiggestellten Neubau wurden Fach- und Sonderlesesäle für Musikdrucke, für Karten und Graphik sowie für Handschriften vorgesehen. Zuvor waren diese Bestände im Altbau und nach dessen Zerstörung auch im Interimsbau im allgemeinen Lesesaal benutzt worden.

Am 1.11.1964 nahm die Musikhistorikerin und Germanistin Dr. Waltraut Linder ihre Tätigkeit als Fachreferentin in der Württembergischen Landesbibliothek auf. Sie hatte zuvor schon seit 1961 Musikhandschriften der WLB im Rahmen des DFG-Programms katalogisiert und kannte die Bibliothek durch ihr anschließendes Referendariat. Sie sollte den Bestandsaufbau für den geplanten Musiklesesaal vorbereiten.

Musikdrucke waren zu diesem Zeitpunkt noch in einer Fachgruppe mit der Grundsignatur Schöne Künste Musiknoten (Sch.K.M.) wie alle alten Fachgruppen alphabetisch aufgestellt. Sie waren nicht im Zuge der Anfang der 1950er Jahre durchgeführten „Aktion Inme"1 mit Individualsignaturen (Fachgruppenbezeichnung mit laufender Nummer) versehen worden.

Nachgewiesen waren die Musikbestände (Musikdrucke und Sekundärliteratur) im alphabetischen Kapselkatalog des Hauses. Da dessen Verfilmung noch nicht in Aussicht stand (sie erfolgte erst 1971), galt es, inn auf Musiktitel durchzusehen und die Titelaufnahmen für den nach dem Krieg begonnenen alphabetischen (PI-)Zettelkatalog der WLB sowie einen alphabetischen und einen Standortkatalog für den späteren Musiklesesaal (auf Matrizen) abzuschreiben und zu vervielfältigen.
Diese von Frau Linder in Angriff genommene Arbeit konnte nur langsam vonstatten gehen, da sie neben ihren weiteren Aufgaben - der Betreuung der neueren Handschriften, dem Baureferat, dem Unterricht in der Bibliotheksschule - bewältigt werden musste.

Zudem musste der Aufbau eines Präsenzbestandes für den zukünftigen Musiklesesaal vorrangig in Angriff genommen werden. Mit in mehreren Raten zugewiesenen Sondermitteln konnten Bestandslücken bei der Sekundärliteratur geschlossen und eine große Sammlung von Taschenpartituren aufgebaut werden, um den gewachsenen Notenbestand um das für eine Musikabteilung unerlässliche klassische Repertoire der Musikliteratur zu ergänzen.

Am 3. August 1970 konnte endlich der Bibliotheksneubau eröffnet werden. Der Musiklesesaal mit drei Abhörkabinen und einem Raum mit einem von

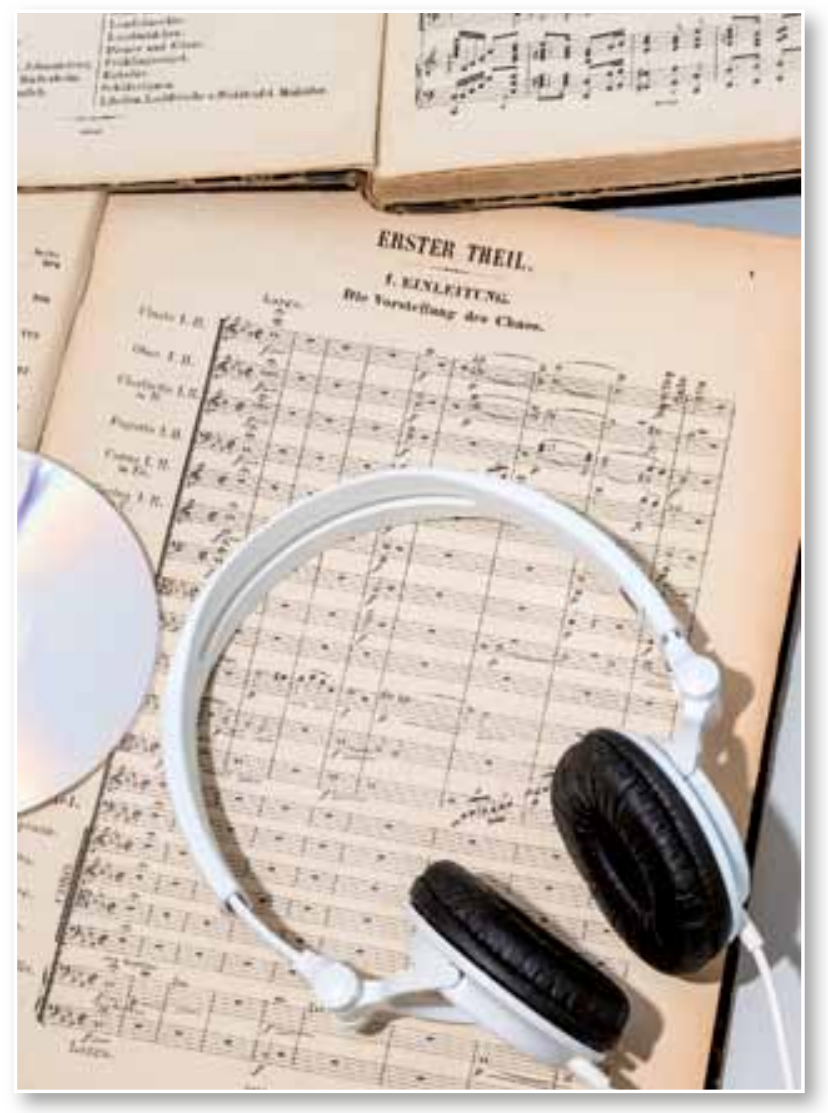

Abb. 1: Noten und Tonträger können im Sonderlesesaal benutzt werden. Im Bild die Partitur der Schöpfung von Joseph Haydn. 
der Stadt Stuttgart zur Eröffnung der Bibliothek geschenkten Steinway-Flügel befand sich - zusammen mit dem Lesesaal für Karten und Graphik bzw. seit 1994 dem Lesesaal für Alte und Wertvolle Drucke - unter dem Hauptlesesaal. Ideal für Benutzer und Mitarbeiter war die Nachbarschaft zur Handschriftenabteilung auf derselben Ebene wegen der inhaltlichen und fachlichen Überschneidungen - bis heute werden Musikhandschriften von dieser verwahrt, betreut und in die Benutzung gegeben. Über eine Wendeltreppe war das ein Stockwerk tiefer gelegene Musikmagazin zu erreichen, in dem die Musikdrucke (Schöne Künste M) Aufstellung fanden.

Für den neuen Musiklesesaal war erstmals eine Planstelle des gehobenen Bibliotheksdienstes bewilligt worden. Unerlässlich für die Arbeit in einer Musikabteilung ist die Fähigkeit, Noten lesen zu können, die nicht ohne Weiteres und nicht ohne praktische Musikausübung erlernt werden kann. Die Stelle konnte mit einer Bibliothekarin besetzt werden, die gerade ihr Examen absolviert hatte, glücklicherweise aus einem musikalischen Elternhaus kam und Klavier spielte.

Erst jetzt konnte die weitere Erschließung und Bearbeitung der Musikdrucke für die Benutzung wieder in Angriff genommen werden. Dazu gehörte das Binden von Kapselschriften, die Vergabe der systematischen freihandtauglichen Signaturen und die Ausstattung der immer noch in der alten Fachgruppe alphabetisch aufgestellten Musikdrucke Schöne Künste $M$ für das Musikmagazin.

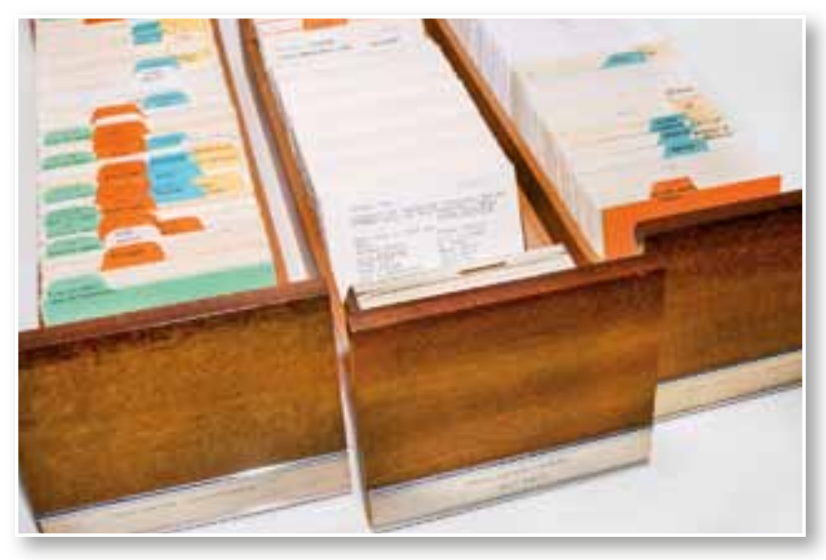

Abb. 2: Katalog von Werken in Sammelbänden (Mitte) sowie Standortkatalog der Musikdrucke: Sammelbände (links) und Werke Johann Sebastian Bachs (rechts).

Die im Haus vorhandene Sekundärliteratur - einschließlich der aus dem Kapselkatalog ergänzten - war im Systematischen Zettelkatalog der WLB (der bis 1995 geführt wurde und heute als DigiSyk in digitaler Form vorliegt) unter der Notation "N" erfasst. Eine Dublette der musikrelevanten Eintragungen des zugehörigen Schlagwortregisters, im Lauf der Zeit erweitert um Verweisungen auf den Standortkatalog der Musikdrucke und auf den Katalog der in Sammelwerken enthaltenen Komponisten, stand zu Auskunftszwecken im Musiklesesaal. Das Schlagwortregister erübrigt sich heute durch die Suchfunktionen der digitalen Version. Der DigiSyk brachte große Vorteile, da nun bei entsprechenden Benutzeranfragen vor Ort im Musiklesesaal vom Fachpersonal Hilfestellung gegeben werden konnte. Vorher konnte man nie sicher sein, ob ein Benutzer den Systematischen Katalog im allgemeinen Katalogsaal und die betreffende Stelle mit der für inn relevanten Literatur, an die man inn verwiesen hatte, tatsächlich finden würde.

Ebenso war es eine große Hilfe, als im Zuge der Verkleinerung der HB Auskunft 1997 alle Bibliographien und die Diskographien des Faches Musik, die dort auf der Empore gestanden hatten, in den Bestand des Musiklesesaals integriert werden konnten.

Im Musiklesesaal befand sich ein Standortkatalog der dort systematisch aufgestellten Werke, der durch ein alphabetisches Schlagwortregister ergänzt wurde. Wie die Kataloge der anderen Lesesäle und Handbibliotheken wurde er 2009 durch die Online-Version "Systematischer Katalog des Musiklesesaals" ersetzt, der im Februar 2015, bedingt durch den Umzug des Bestandes auf die Empore des Hauptlesesaals, in "Systematischer Katalog Musik" umbenannt wurde. Durch die Suchfunktionen der Online-Version erübrigt sich auch hier das alphabetische Schlagwortregister.

Der (alte) alphabetische (PI-)Katalog des Musiklesesaals, der heute in digitalisierter Form als "Musik-AK" im DigiKat enthalten ist, sollte alle Musikdrucke und Sekundärliteratur der WLB möglichst umfassend nachweisen. Er verzeichnet Literatur bis Erscheinungsjahr 1989 und wurde für Literatur ab Erscheinungsjahr 1990 vom neuen alphabetischen (RAK-)Katalog und später vom Online-Katalog ${ }^{2}$ abgelöst. Für die nun erforderliche Katalogisierung nach den Sonderregeln RAK-Musik 
konnte 1989 eine Bibliothekarin mit musikbibliothekarischem Zusatzstudium ${ }^{3}$ für den Musiklesesaal eingestellt werden.

Im Aufbau befanden sich zwei Kataloge: der (systematische) Standortkatalog der Musikdrucke und der sogenannte Besetzungskatalog. Der Standortkatalog war Voraussetzung für den Zutritt der Benutzer ins Musikmagazin und für die Signaturvergabe. Er befindet sich heute als Zettelkatalog im Musikmagazin und ist nach wie vor für bestimmte Suchanfragen ${ }^{4}$ sowie zum Überprüfen und Revidieren unentbehrlich. Der Besetzungskatalog ist systematisch nach musikalischen Besetzungen (Orchester, Singstimme und Klavier etc.) sowie Formen und Gattungen (Sinfonien, Messen, Arien etc.) geordnet; er liegt in digitalisierter Form als DigiSyk Musik vor. ${ }^{5}$

Der DigiSyk Musik wurde abgelöst von der kooperativen Sacherschließung musikalischer Werke, die seit 2004 erfolgreich im SWB Anwendung findet (Stand 9. Januar 2015: 224.409 Titelsätze mit dem eigens für die Sacherschließung Musik eingerichteten Feld 5590). Diese wurde in einer speziell dafür ins Leben gerufenen SWB-AG von der WLB, der Pfälzischen Landesbibliothek Speyer (bis zu ihrem Wechsel zum HBZ) sowie den Bibliotheken der fünf Musikhochschulen Baden-Württembergs und der Musikhochschule Leipzig entwickelt als Weiterführung der früher lokal geführten Zettelkataloge mit zum Teil hausinternen Regeln. Die Schlagwortfolgen sind noch nicht im aDIS-OPAC recherchierbar, für die Recherche wird auf den Musik-OPAC des SWB zurückgegriffen, wo per Bibliothekssigel auf die Bestände der WLB eingeschränkt werden kann. Außer der Suche nach einzelnen Schlagwörtern ist hier überdies noch die Suchmöglichkeit über ein Schlagwortfolgenregister gegeben. Im derzeitigen RDA-Projekt versucht eine Themengruppe der UAG Musik der

3) Eine solche Zusatzausbildung an der damaligen Fachhochschule für Bibliothekswesen Stuttgart, heute Hochschule der Medien (HdM) Stuttgart, gab es zuvor lange Zeit nur für Bibliothekare Öffentlicher Bibliotheken

4) Beispielbände zur Musikgeschichte, weitere Sammelbände und die Werke der einzelnen Komponisten sind nach Besetzung, Gesangbücher chronologisch und Volkslieder nach Ländern und Regionen verzeichnet und aufgestellt.

5) Da dies besonders auch für den praktischen Musiker von großer Bedeutung ist, sind Musica practica und Tonträger in Öffentlichen und Musikhochschulbibliotheken grundsätzlich so aufgestellt und präsentiert. So spiegelt sich dieser Erschließungsaspekt auch in der systematischen Aufstellung des Musikmagazins wider.

6) Der Benutzer sucht ein bestimmtes Musikstück. Es ist ihm gleichgültig, in welchem Sammelwerk es enthalten ist.
AG RDA eine überregionale bzw. internationale Erschließung der musikalischen Besetzung und der musikalischen Form/Gattung als Ablösung der lokalen Insellösungen der einzelnen Bibliotheken durchzusetzen.

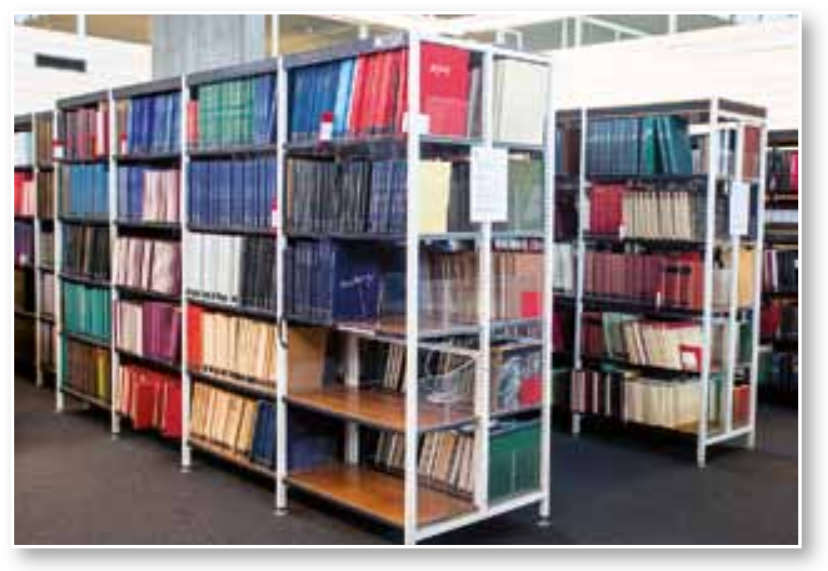

Abb. 3: Lesesaal Musik auf der Empore des Hauptlesesaals

Als Ergänzung zum alphabetischen Katalog wurde im Lauf der Zeit ein alphabetisch nach Komponistennamen geordneter Zettelkatalog zur Erschließung der in Sammelwerken und -bänden enthaltenen Werke, die sogenannte A-Reihe, aufgebaut und geführt. Wegen eingeschränkter Arbeitskapazität wurden nur die Komponistennamen ohne die Titel der enthaltenen Werke aufgeführt, was in der Praxis dennoch relativ gut funktioniert(e), da sich bereits viel am Titel des Sammelwerkes ablesen lässt, beispielsweise kann in einem "Liederbuch des Schwäbischen Albvereins" (Notenausg. von Karl und Hellmut Aichele) keine Kantate oder Sinfonie enthalten sein. Nur unter Hinzuziehung dieses Kataloges ist es möglich, alle in der WLB vorhandenen Werke eines Komponisten vollständig nachzuweisen unabhängig davon, ob es sich um einen Komponisten von regionaler Bedeutung wie z.B. Friedrich Silcher, Emilie oder Johann Rudolf Zumsteeg oder um einen generell bedeutenden Komponisten wie z.B. Wolfgang Amadeus Mozart handelt. Später bot sich eine komfortablere und weniger aufwendige Erfassung unselbständiger Werke nach RAK(-Musik) und damit die Verzeichnung im Online-Katalog an. ${ }^{6}$ Der Zettelkatalog befand sich im Musiklesesaal. Bei dessen Schließung konnte er nur im Musikmagazin untergebracht werden und ist seither für Benutzer nicht mehr zugänglich. 
Eine historische Erschließung der Komponisten war gewährleistet durch die chronologische Anlage der Musikgeschichte (NF) im Systematischen Zettelkatalog der WLB (heute DigiSyk) sowie durch die chronologische Aufstellung der Literatur über die einzelnen Komponisten ( $\mathrm{Ne}$ ) und ihrer Gesamtausgaben (Nq) im Lesesaal Musik, wo sie für die dort stehenden Bestände bis heute weitergeführt ist.

Eine Erschließung nach Stoffen, Motiven u.ä. war vorgesehen, konnte aber nie verwirklicht werden. Wenigstens stehen heute einige Speziallexika zu dieser Thematik zur Verfügung. ${ }^{7}$

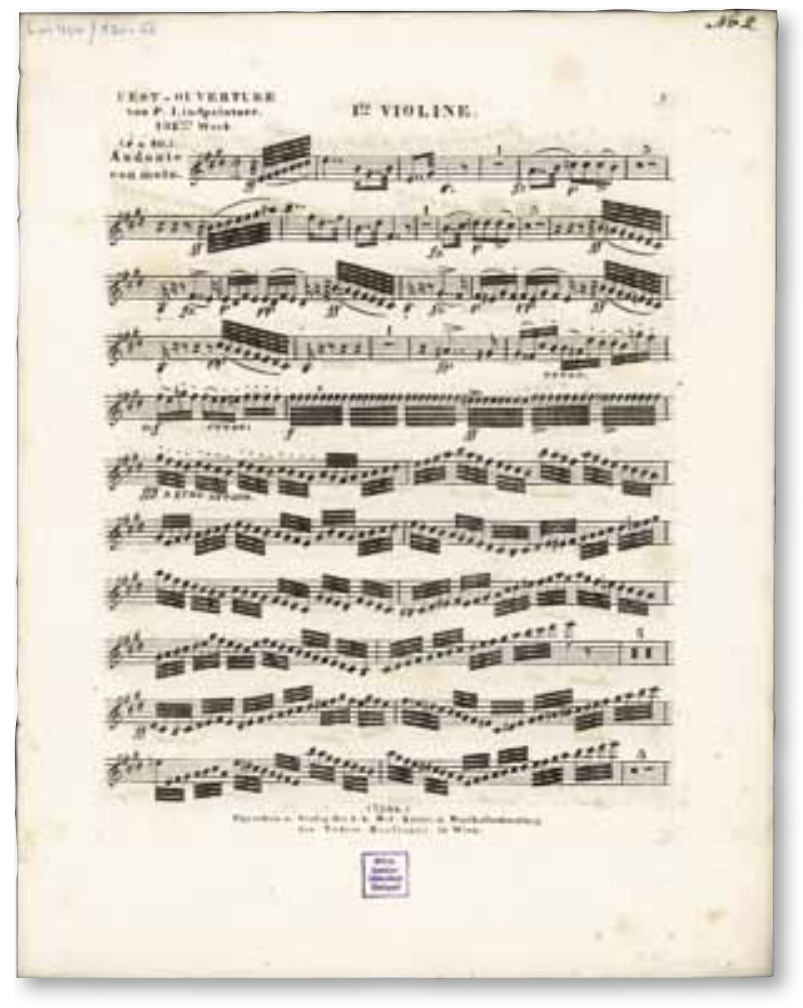

Abb. 4: Fest-Ouverture (in E dur) für das ganze Orchester. Opus 102 I Peter Lindpaintner. [Stimme der 1. Violine]. Wien: Haslinger [184.]

Im Vergleich zu anderen großen Musiksammlungen war und ist der Musiklesesaal der WLB mit einer (1970-1989) und später eineinhalb (1989-1991) bzw. zwei Planstellen (ab 1991) für den gehobenen Dienst personell wesentlich geringer ausgestattet. Deshalb leistete Frau Linder u.a. viel Auskunftsdienst im Musiklesesaal, zumal immer wieder längere krankheitsbedingte personelle Engpässe zu verkraften waren und seit dem Umzug der Abteilung Karten und Graphik 1994 in die Gaisburgstraße auch der dort tätige Bibliothekar nicht mehr als Vertretung zur Verfügung stand. Mit seinem Wissen in sehr speziellen Bereichen der Musik übernahm er bis zu seinem Eintritt in den Ruhestand (2007) immer wieder beratende Funktion bei bestimmten Anfragen und tut dies darüber hinaus bis auf den heutigen Tag.

So beschäftigte der Aufbau dieser Kataloge Frau Linder im Grunde bis zu ihrem Eintritt in den Ruhestand, der sich um ein Jahr verzögerte, da der erste als Nachfolger vorgesehene Referendar die Ausbildung abbrach. Der tatsächlich nachfolgende Fachreferent konnte nach seinem Referendariat in der WLB 1993 auf dem Bestehenden sowie einer funktionierenden Abteilung aufbauen und sich weiteren Arbeiten wie Ausstellungen, Veröffentlichungen und der Vernetzung widmen.

Unter seiner Ägide entstanden - mit den neuen Möglichkeiten der EDV, die inzwischen Einzug gehalten hatte - verschiedene Datenbanken: ${ }^{8}$ eine Datenbank der in der Handschriftenabteilung aufbewahrten Musik- und Musikerhandschriften; ein Verzeichnis der musikalischen Nachlässe und Sammlungen sowie die aus den Theaterzetteln der Württembergischen Staatstheater generierten Datenbanken des Musiktheaters (Opern, Ballette, Divertissements, Pantomimen, Tänze), der Abonnementskonzerte und des Repertoires. Andere Verzeichnisse und Datenbanken wurden von verschiedenen Bearbeitern (teilweise in der WLB und teilweise extern) erarbeitet, so z.B. der Katalog der Gesangbücher in Württemberg (mit Online-Nachträgen) oder die Josephine-Lang-Datenbank. ${ }^{9}$

Kooperation zwischen den Sammlungen mit relevanten Musikbeständen gibt es seit vielen Jahren. Ideal waren an der "Kulturmeile" die unmittelbare Nachbarschaft zur Musikabteilung der Stadtbibliothek (bis zu deren Umzug in das neue Gebäude hinter dem Hauptbahnhof im Herbst 2011) und zur Bibliothek der Musikhochschule.

1995 wurde die "Arbeitsgemeinschaft Stuttgarter Musikbibliotheken“ ins Leben gerufen, ${ }^{10} z u$ der im Lauf der Zeit weitere Bibliotheken im Umland stießen. Derzeit gehören ihr neben der WLB die Stadt-

7) Die dargestellten Erschließungsarten des Musikbestands sind Standard in Musikbibliotheken und orientieren sich an den Bedürfnissen der Benutzer.

8) Sie sind alle auf den Webseiten der Musiksammlung verlinkt: http:// www.wlb-stuttgart.de/sammlungen/musik/ [2015-08-31].

9) Die online vorliegenden Verzeichnisse und Datenbanken sind ebenfalls auf den Webseiten der Musiksammlung verlinkt.

10) Die erste Sitzung fand am 15.12.1995 in der Bachakademie statt. 
bibliotheken Heilbronn, Reutlingen, Stuttgart und UIm, die Bibliotheken der Hochschule für Musik und Darstellende Kunst Stuttgart sowie der Hochschulen für Kirchenmusik Rottenburg und Tübingen, die Landeskirchliche Zentralbibliothek, die Bibliothek der Internationalen Bachakademie Stuttgart, die Bibliotheken des Südwestrundfunks und der Staatstheater Stuttgart sowie die ekz bibliotheksservice $\mathrm{GmbH}$ an.

Die bundesweite (und internationale) Vernetzung ist innerhalb der Internationalen Vereinigung der $\mathrm{Mu}$ sikbibliotheken, Musikarchive und Dokumentationszentren (IVMB) gegeben, deren deutsche Gruppe wegen der Gründung 1951 in Paris den französischen Namen Association Internationale des Bibliothèques, Archives et Centres de Documentation Musicaux (AIBM) verwendet. Die jährlichen Tagungen dienen dem fachlichen Austausch und der Fortbildung.

Die zweite Nachfolgerin Frau Linders führte die Benutzung von Tonträgern ein, die bis dahin, von denen des Hölderlin-Archivs abgesehen, lediglich Archivbestand waren. Grund war, dass es sich (bis auf wenige zu vernachlässigende Beilagen zu Büchern oder Zeitschriften u.ä.) um eine heterogene Sammlung von Pflichtexemplaren handelt, die Stadtbibliothek Stuttgart dagegen für die Region Stuttgart umfassend sammelt. Als seit Ende der 80er Jahre die so einfach und kostengünstig herstellbaren Compact-Discs abgeliefert wurden, überstieg die Katalogisierung die Kapazitäten des Musiklesesaals. Erst 2004 begann eine Bibliothekarin, die seit 2006 als Nachfolgerin der ersten Bibliothekarin im Musiklesesaal tätig ist und 2008 die Zusatzausbildung Musikinformationsmanagement ${ }^{11}$ an der Hochschule der Medien Stuttgart absolvierte, mit der retrospektiven Erschließung.

1996 wurden für einen Teil der Neuzugänge an Musikdrucken Numerus-currens-Signaturen (Signatur-Kontingent ../100000) und deren Aufstellung im allgemeinen Magazin eingeführt. Von dort werden sie beim Lesesaaldienst angefordert. Dies bedeu-

Abb. 5: Titel mit enthaltenen Werken und Sacherschließung Musik im Musik-OPAC des SWB, vgl. Abschnitte "Angaben zum Inhalt" bzW. "Besetzung, Gattung (Musik)"

tete Zeitersparnis gegenüber der relativ aufwendigen Vergabe der Signaturen für das Musikmagazin, die wegen der Bedeutung des Werk- oder Einheitssachtitels für die systematische Aufstellung noch dazu nach oder in Zusammenhang mit der Katalogisierung erfolgen musste. In der Benutzung ist zu beachten, dass genau diese Neuausgaben im Online-Katalog, auf den die Benutzer als aktuellen Katalog gelenkt werden, nachgewiesen sind. Die im Musikmagazin stehenden Musikdrucke, die im Musiklesesaal durch das Auskunftspersonal per Sofortausleihe erhältlich waren, sind dagegen nur im DigiKat (Musik-AK) nachgewiesen. Seit 2004 erhalten alle Neuzugänge von Musikdrucken eine Numerus-currens-Signatur, da mit der Einstellung des Zetteldrucks der nur in Zettelform existierende Standortkatalog des Musikmagazins nicht mehr weitergeführt werden konnte und deshalb die systematische Aufstellung im Musikmagazin abgebrochen wurde.

\section{2 wurden die Auskunftstellen für Alte und} Wertvolle Drucke und für Kunst zusammengelegt. Deshalb zog der Musiklesesaal in den bisherigen Kunstlesesaal ein Stockwerk tiefer um. Die Flügelund Handschriftenbenutzung erfolgte nach wie vor am alten Platz. Für die katalogisierende Mitarbeiterin, die bis dahin wegen der notwendigen Verfüg- 
barkeit der Nachschlagewerke zur Ermittlung von Einheitssachtiteln u.ä. einen offenen Arbeitsplatz im Musiklesesaal hatte und nun ein Zimmer daneben erhielt, war der Umzug ein Vorteil.

Im Februar 2015 wurde der Musiklesesaal geschlossen, da der Trakt, der ihn sowie den Vortragsraum, das Zimmer der Württembergischen Bibliotheksgesellschaft und den Aufenthaltsraum für die Mitarbeiter der WLB beherbergte, wegen des Erweiterungsbaus abgerissen werden muss te. Die derzeitige Leiterin der Musiksammlung hatte die Aufgabe, die Schließung des Musiklesesaals vorzubereiten und den Umzug der Präsenzbestände, die künftige Benutzung (auch von Musikdrucken und Tonträgern) sowie die Einbindung der beiden Mitarbeiterinnen des Musiklesesaals in Haupt- und Sonderlesesaal zu organisieren und durchzuführen. Musikdrucke und Tonträger werden nun im Sonderlesesaal ${ }^{12}$ benutzt, dessen Öffnungszeit von 10-17 Uhr derjenigen des bisherigen Musiklesesaals entspricht. Der begleitend zu benutzende, sehr aktuell gehaltene Präsenzbestand befindet sich im "Lesesaal Musik" auf der Empore des Hauptlesesaals, wo die längere Öffnungszeit von 8-20 Uhr ${ }^{13}$ von Vorteil ist. ${ }^{14}$

Als Fachpersonal sind nach wie vor die beiden Mitarbeiterinnen des Musiklesesaals auf den Webseiten der Musiksammlung als Ansprechpartnerinnen für Musikanfragen genannt. Ihre Erreichbarkeit ist wegen Sonder- und Hauptlesesaaldiensten, Nachschlagen im Präsenzbestand u.ä. am ehesten per E-Mail gewährleistet. Die der Fachwelt ohnehin bekannte Bedeutung der Musiksammlung ist durch die Webseiten der WLB und andere Möglichkeiten im Bewusstsein der Öffentlichkeit vermittelbar. Dennoch bleibt zu hoffen, dass nach Bezug des Erweiterungsbaus die Musikbestände wieder zusammengeführt werden können. ${ }^{15}$

Martina Rommel

\section{Literaturverzeichnis:}

Die Musiksammlung / [Waltraut Linder] // In: Württembergische Landesbibliothek Stuttgart / verf. und zsgest. von Mitarb. der Württembergischen Landesbibliothek. - Stuttgart : Württembergische Landesbibliothek, 1971. - 104 S. : III. - S. $66-71$.

Musikbibliothekarische Städteporträts : Stuttgart // In: Forum Musikbibliothek. -1980 , H. 2, S. $44-82$.

Der Musiklesesaal [und] Die Musiksammlung / [Waltraut Linder ] // In: Württembergische Landesbibliothek Stuttgart : ein Führer durch ihre Geschichte und ihre Sammlungen / [Württembergische Landesbibliothek. Red.: Horst Hilger .... . - Stuttgart : Württembergische Landesbibliothek, 1990. - 140 S. : III. - ISBN 3-88282-026-8. - S. 64 - 65 [und] S. 93 - 97.

Württembergische Landesbibliothek : Notenschätze ; die Bestände der Musikabteilung // In: Stuttgarter Zeitung. - Nr. 14, 19.1.1971.

Musica practica in der Württembergischen Landesbibliothek : aus der Geschichte ihrer Bestände / Waltraut Linder // In: Wolfenbütteler Notizen zur Buchgeschichte. - 11 (1986), S. 116 - 141.

Geschichte der Württembergischen Landesbibliothek / von Karl Löffler. Nachdr. der Ausg. Leipzig 1923. - Nendeln : Kraus, 1968. - IV, 262 S.

Malo, Markus: Die Kataloge der Königlichen Öffentlichen Bibliothek und der Württembergischen Landesbibliothek Stuttgart. - Stuttgart : Württembergische Landesbibliothek, 2002. - 53 BI. : III. ; $30 \mathrm{~cm}$. - ISBN 3-88282-058-6.
12) Der Sonderlesesaal befindet sich im ursprünglichen Musiklesesaal und dient inzwischen der Benutzung von Sonderbeständen der Bereiche Alte und Wertvolle Drucke, Handschriften, Karten und Graphik, Kunst und Musik. Das Musikmagazin ist allerdings nicht mehr über die einstige Wendeltreppe zu erreichen, da diese im Zuge der Zusammenlegung der Abteilungen Alte und Wertvolle Drucke und Kunst abgebaut wurde.

13) Samstags sind beide Lesesäle von 10-13 Uhr geöffnet.

14) Ein Tausch mit dem Präsenzbestand Kunst war in der Kürze der für die Umzugsvorbereitungen zur Verfügung stehenden Zeit nicht möglich.

15) Für einige Einzelhinweise danke ich Kolleginnen und Kollegen der WLB und der AG Stuttgarter Musikbibliotheken. Mein besonderer Dank gebührt jedoch Ingeborg Krekler für die Klärung zahlreicher Fragen. 